\title{
Harmonization of a multi-sensor navigation system
}

\author{
Eric Dorveaux and Nicolas Petit \\ MINES-ParisTech / Département Mathématiques et Systèmes / Centre Automatique et Systèmes (CAS), Paris, France. \\ Email: \{eric.dorveaux I nicolas.petit\}@ mines-paristech.fr
}

\begin{abstract}
We address the problem of harmonization of two subsystems used separately to measure a velocity in a body reference frame and an attitude with respect to an inertial frame. This general problem is of particular importance when the two subsystems are jointly used to obtain an estimate of the inertial velocity, and, then, the position through an integration procedure. Typical possible applications encompass pedestrian navigation, and, more generally, indoor navigation. The paper demonstrates how harmonization can be achieved thanks to an analysis of propagation of projection errors. In particular, trajectories known to be closed can be used to determine one dominant harmonization factor which is the pitch relative orientation angle between the two subsystems. Computations yielding the harmonization procedure are exposed in this article, and experimental data serve to illustrate the application of the proposed method.
\end{abstract}

Keywords-Indoor Positioning, Harmonization, Calibration, Path Reconstruction, Inertial Sensor.

\section{INTRODUCTION}

Recently, numerous multi-sensors systems for indoor navigation have been proposed. Among the currently studied technologies, several rely on radio signals from preinstalled infrastructures like iGPS, WLan, Wifi and Ultra Wide Band (UWB), and some others rely on various kinds of beacon, like ultra sounds systems or fingerprinting techniques. Alternatively, infrastructure-free techniques are also studied, like vision-based systems or systems using inertial navigation such as foot-mounted inertial measurement unit (IMU). Several of these technologies are often combined. The benefits of simultaneously using multiple sensors are essentially twofolds: i) various type of information can be obtained, ii) the overall robustness of the information obtained through data reconciliation is largely improved. While being practically very effective, these techniques also have flaws. A key aspect of these complex embedded systems is that information from the various sources must be harmonized, i.e. some procedure is necessary to make the informations consistent. While of importance, this aspect is often underestimated, even by practitioners. We address it in this paper.

Among the sources of inconsistencies between the sensors are individual sensor ill-calibration, and relative misalignments of their frames of references. Individually, each sensor has its own defects. Fortunately, numerous methods can be used to calibrate them. These methods are usually sufficient to determine scale factors and biases ([1], [2], [6], [8]). For this reason, in this paper, we assume that the sensors themselves are already calibrated and we consider the problem of sensors harmonization.

The setup we consider in this study is a positioning system consisting of an inertial velocity estimator whose output signal is integrated over time. Two sources of information are used to obtain this estimate: a body velocity estimate and an

978-1-4577-1804-5/11/\$26.00 (C)2011 IEEE attitude sensor. This set-up covers a large class of navigation systems. A first example is a velocimeter and a compass used to estimate the position of a wheeled robot (e.g. Pioneer IV Mobile Robots). A second example is a visual (camerabased) odometer used in conjunction with an IMU for attitude estimate ([5], [7]). A third application is a body mounted laser or Doppler radar used with an IMU attitude sensor ([4]). From a theoretical viewpoint, such systems use an estimate of the body velocity, expressed in the reference frame of the sensors providing this information, and an attitude estimate, expressed in the reference frame of the IMU. Assuming that all the sensors are attached to a common rigid body, there exists a constant rotation matrix (referred to as the harmonization matrix in this article) between the two considered frames of reference. In general, this matrix is not the identity matrix, and when it is ignored when determining a velocity with respect to the inertial frame of reference, the process results in projection errors. As the errors are integrated over time, the reconstructed trajectories drift.

The paper proposes a method to compensate for such drift. Notations and the problem statement are presented in Section I. In Section II, an analytic study exposes the sources of the problem, and shows that the harmonization matrix can be identified by a simple experimental procedure. The discussed pedestrian application is considered for sake of illustration. The main result shows that the dominant terms in the harmonization matrix (the pitch angle) can be determined by analysis of the vertical drift observed along a closed curve. Experimentally, this result can be very conveniently exploited in an on-the-field calibration procedure, where the user carrying the considered positioning system is simply asked to walk along a closed curve of his choice. At his return point, the harmonization matrix is automatically calculated, which permits to calibrate the multi-sensor system. These practical topics are presented in Section III. Finally some experimental results are presented in Section IV.

\section{Problem Statement \& Notations}

The problem we wish to address is to estimate the harmonization matrix (totally or at least its most impacting terms) between the frame where the velocity is measured or estimated and the one in which the attitude is known.

Consider the following systems, each being attached to a coordinate frame:

- a building considered as the inertial frame of reference $\Re_{I}$ (with its horizontal plane denoted $\mathrm{x}-\mathrm{y}$ and $\mathrm{z}$ pointing upward);

- a pedestrian moving in the building with an attached frame $\Re_{\text {ped }}$ whose $\mathrm{x}$ and $\mathrm{y}$-axis are horizontal and $\mathrm{x}$ is pointing forward;

- a subsystem providing an inertial speed expressed in its own coordinate frame $\Re_{v e l}$; 
- an IMU providing the attitude of its own coordinate frame $\Re_{I M U}$ with respect to the inertial frame of reference $\Re_{I}$. The attitude is initialized such that the gravity is on the vertical axis ${ }^{1}$.

Indices ped, vel, $I M U$ and $I$ refer to which frame the corresponding quantity is expressed in. For instance, $V_{\text {vel }}$ is the speed expressed in $\Re_{v e l}$, whereas $V_{I M U}$ is the same speed expressed in $\Re_{I M U}$. Figure 1 pictures the introduced coordinate frames and indicates the rotation matrices between them. The ones of interest are:

- $R_{\text {plane }}=R_{\text {ped } \rightarrow I}$, rotation matrix from the pedestrian frame to the inertial frame (unknown)

- $R_{b o d y}^{T}=R_{p e d \rightarrow v e l}$, rotation matrix from the pedestrian frame to the velocity sensor frame (unknown)

- $R_{\text {harmo }}=R_{v e l \rightarrow I M U}$, rotation matrix from the velocity sensor frame to the IMU frame (unknown, to be identified)

- $R_{a t t}=R_{I M U \rightarrow I}$, rotation matrix from the IMU frame to the inertial frame (measured by the IMU).

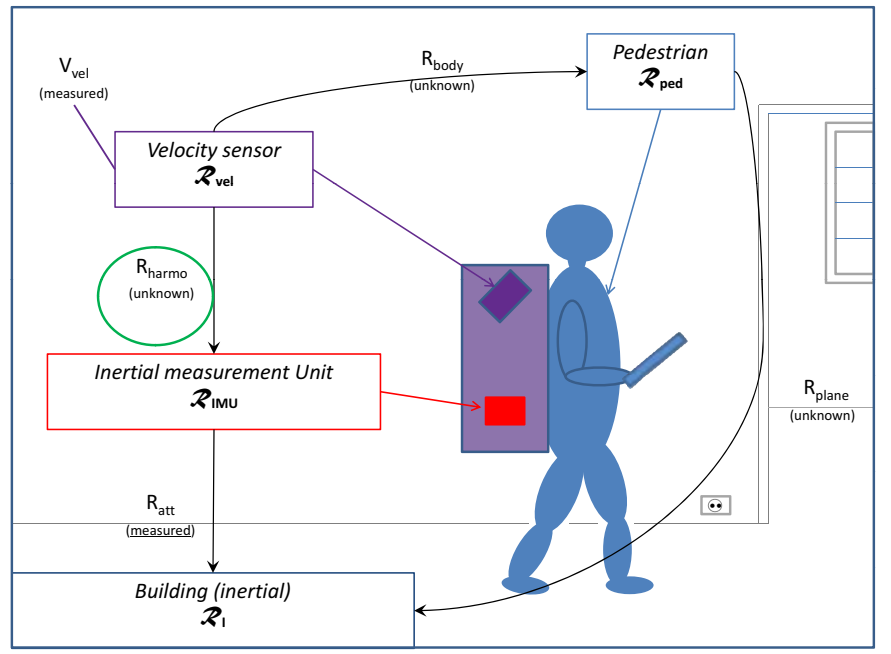

Fig. 1. Diagram representing the various coordinate frames and rotation matrices of interest. The speed $V_{v e l}$ is measured in the velocity frame $\Re_{v e l}$. The rotation matrix $R_{I M U \rightarrow I}=R_{a t t}$ is measured. The rotation matrix $R_{\text {harmo }}$ is to be identified.

The following useful relations hold

$$
\begin{aligned}
V_{I} & =R_{\text {att }} R_{\text {harmo }} \underbrace{V_{\text {vel }}}_{\text {speed which is measured }} \\
V_{\text {vel }} & =R_{\text {body }}^{T} V_{\text {ped }} \\
\text { and } \quad I_{3} & =R_{\text {att }} R_{\text {harmo }} R_{\text {body }}^{T} R_{\text {plan }}^{T}
\end{aligned}
$$

which yields $\quad V_{I}=R_{\text {plan }} R_{\text {body }} V_{\text {vel }}$

The most interesting frame to express the speed in is the inertial frame $\Re_{I}$. Indeed, in this frame, it is possible to integrate it to obtain the trajectory of the pedestrian in the building. However, the speed is measured in the velocity frame $\Re_{v e l}$. The matrix $R_{b o d y}$ is unknown and is relatively uncertain because, each time the pedestrian uses the navigation system, it could be put into a slightly different orientation. Finally, $R_{\text {plane }}$ is unknown as well. Therefore, the easiest way to express the speed in the inertial frame is to determine the

\footnotetext{
1 The yaw angle is left free to allow a later absolute alignment (e.g. using a map if one is available).
}

harmonization matrix $R_{\text {harmo }}$ between the velocity frame and the IMU-frame, and thus exploit Equation (1) and not Equation (2). This matrix depends only on the relative orientations of the two sensor subsystems. The positions and orientations of the subsystems being constant, $R_{\text {harmo }}$ needs to be identified only once and for all.

The relative position at any current time $T, P(T)-P(0)$, with respect to an inertial position $P(0)$ is given through

$$
P(T)=P(0)+\int_{0}^{T} R_{a t t} R_{\text {harmo }} V_{\text {vel }} d t
$$

Ideally, the harmonization matrix $R_{\text {harmo }}$ should be close to identity, but errors of a few degrees cannot be avoided in the actual set-up of the subsystems when off-the-shelf packaged sensors are used. In the following, we model the impact on trajectory reconstruction when this matrix is omitted. Then, we show how to compensate for this error.

\section{IDENTIFICATION AND CORRECTION OF TRAJECTORY ERRORS DUE TO AN HARMONIZATION ERROR}

\section{A. Trajectory errors: the shape of the trajectory is altered}

For sake of illustration, we consider a particular case where the pedestrian is walking with a speed of constant norm, and, for simplicity, in the forward direction (i.e. along the $\mathrm{x}$-axis of $\left.\Re_{\text {ped }}\right)$. First, we assume that the pedestrian frame, the velocity frame and the IMU frame are identical, i.e. that $R_{\text {harmo }}=$ $R_{\text {body }}=I_{3}$. So, we have

$$
V_{p e d}=V_{v e l}=V_{I M U}=\left(\begin{array}{c}
V_{0} \\
0 \\
0
\end{array}\right)
$$

Further, we assume that the pedestrian is walking in an horizontal plane over the time interval $[0, T]$. The IMU attitude is thus expressed using a single angle $\alpha(t)$

$$
R_{a t t}(t)=\left(\begin{array}{ccc}
\cos (\alpha) & -\sin (\alpha) & 0 \\
\sin (\alpha) & \cos (\alpha) & 0 \\
0 & 0 & 1
\end{array}\right)=R(\alpha(t))
$$

Through Equation (3), one gets

$$
\begin{aligned}
P(T) & =P(0)+\int_{0}^{T} R(\alpha(t)) \cdot V_{I M U} \cdot d t \\
& =P(0)+V_{0} \int_{0}^{T}\left(\begin{array}{c}
\cos (\alpha) \\
\sin (\alpha) \\
0
\end{array}\right) \cdot d t
\end{aligned}
$$

Now, assume that the pedestrian has walked along a closedcurve. Then, $P(T)=P(0)$. From the previous equality, we deduce that ${ }^{2}$.

$$
\int_{0}^{T} \cos (\alpha(t)) d t=\int_{0}^{T} \sin (\alpha(t)) d t=0
$$

Now, we introduce an harmonization error, i.e. there is now a rotation $R_{\text {harmo }} \neq I_{3}$ between the coordinate frame where the speed is measured in $\left(\Re_{v e l}\right)$ and the one where the attitude is measured in $\left(\Re_{I M U}\right)$. We split up $R_{\text {harmo }}$ into

\footnotetext{
${ }^{2}$ One shall note that this last property is true even if the velocity $V_{I M U}$ is not aligned with the $\mathrm{x}$-axis but belongs to the $x-y$ plane (see Section III).
} 
three successive canonical constant rotations (roll $\phi$, pitch $\theta$, and yaw $\psi$ in this order from $\Re_{I M U}$ to $\Re_{v e l}$ ) and note $R_{\text {harmo }}^{T}$

$\left(\begin{array}{ccc}c(\psi) & -s(\psi) & 0 \\ s(\psi) & c(\psi) & 0 \\ 0 & 0 & 1\end{array}\right)\left(\begin{array}{ccc}c(\theta) & 0 & s(\theta) \\ 0 & 1 & 0 \\ -s(\theta) & 0 & c(\theta)\end{array}\right)\left(\begin{array}{ccc}1 & 0 & 0 \\ 0 & c(\phi) & -s(\phi) \\ 0 & s \phi) & c(\phi)\end{array}\right)$

Assume that the velocity frame $\Re_{v e l}$ has been slightly rotated. The speed $V_{I M U}=\left(\begin{array}{lll}V_{0} & 0 & 0\end{array}\right)^{T}$ in the IMU-frame is unchanged, but the speed measured in the velocity frame $\Re_{v e l}$ now writes

$$
\begin{aligned}
V_{\text {vel }} & =R_{\text {harmo }}^{T}\left(\begin{array}{c}
V_{0} \\
0 \\
0
\end{array}\right) \\
& =V_{0}\left(\begin{array}{c}
c(\theta) \cdot c(\psi) \\
c(\theta) \cdot s(\psi) \\
-s(\theta)
\end{array}\right) \\
& \neq V_{I M U}
\end{aligned}
$$

If the harmonization error is not taken into account in the trajectory reconstruction, one obtains

$$
\begin{aligned}
& P_{r}(T)-P(0)=\int_{0}^{T} R_{a t t}(t) V_{\text {vel }} \cdot d t \\
& =V_{0} \cdot \int_{0}^{T}\left(\begin{array}{ccc}
c(\alpha(t)) & -s(\alpha(t)) & 0 \\
s(\alpha(t)) & c(\alpha(t)) & 0 \\
0 & 0 & 1
\end{array}\right)\left(\begin{array}{c}
c(\theta) \cdot c(\psi) \\
c(\theta) \cdot s(\psi) \\
-s(\theta)
\end{array}\right) \cdot d t \\
& =\left(\begin{array}{c}
T \\
0 \\
-T \cdot V_{0} \cdot \sin (\theta)
\end{array}\right)^{T} \neq 0=P(T)-P(0)
\end{aligned}
$$

where the vector $(c(\theta) \cdot c(\psi) \quad c(\theta) \cdot s(\psi) \quad-s(\theta))^{T}$ has been put outside the integral since it is constant.

According to Equation (7), the reconstructed position will then slightly, but constantly, drift downward (or upward depending on the signs of $\theta$ and $V_{0}$ ) as illustrated in the simulation presented in Figure 2. In Figure 3, the same drift can be observed on experimental data obtained with the system briefly presented in Section IV. As a consequence, even if closed paths are followed, the estimated trajectory will not go back to the starting point as it should. Interestingly, one can note that the two other angles have no impact on Equation (7). Only the pitch angle $\theta$ alters the shape of the reconstructed trajectory. The trajectory will simply be rotated if the yaw angle is not zero (as illustrated in Figure 2 when only the pitch angle is corrected). The roll angle does not have any effect since the movement is performed along its rotation axis. Note that the error in Equation (7) is proportional to the traveled distance $V_{0} \cdot T$. An angle as small as 3 degrees leads to an error of more than $5 \%$ of the traveled distance.

\section{B. First proposed calibration procedure}

In Section II-A, we have explained how even a small pitch angle error could alter the shape of the trajectory at a macroscopic scale. A first calibration procedure is proposed in this section. It takes advantage of this property. From the measurements, both the traveled distance $V_{0} \cdot T$ and the reconstructed trajectory neglecting the harmonization matrix (Equation (6)) are easily computable. They lead to the following simple calibration procedure:

1) Walk along any closed-loop trajectory in the horizontal plane with the following constraints: the speed direction

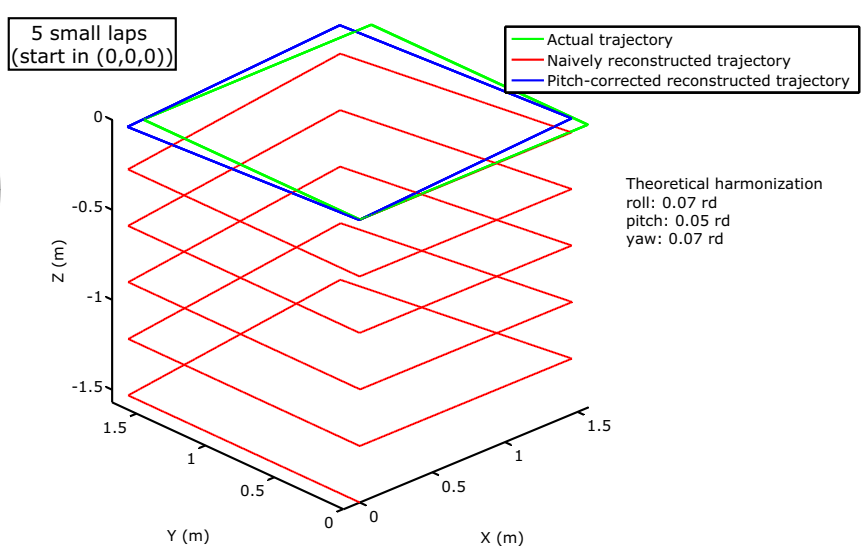

Fig. 2. Simulation results - The harmonization angles are given on the right of the figure. Neglecting the harmonization, even for low pitch angles, can generate important drifts. Performing the harmonization with only a pitchangle eliminates the closure error.

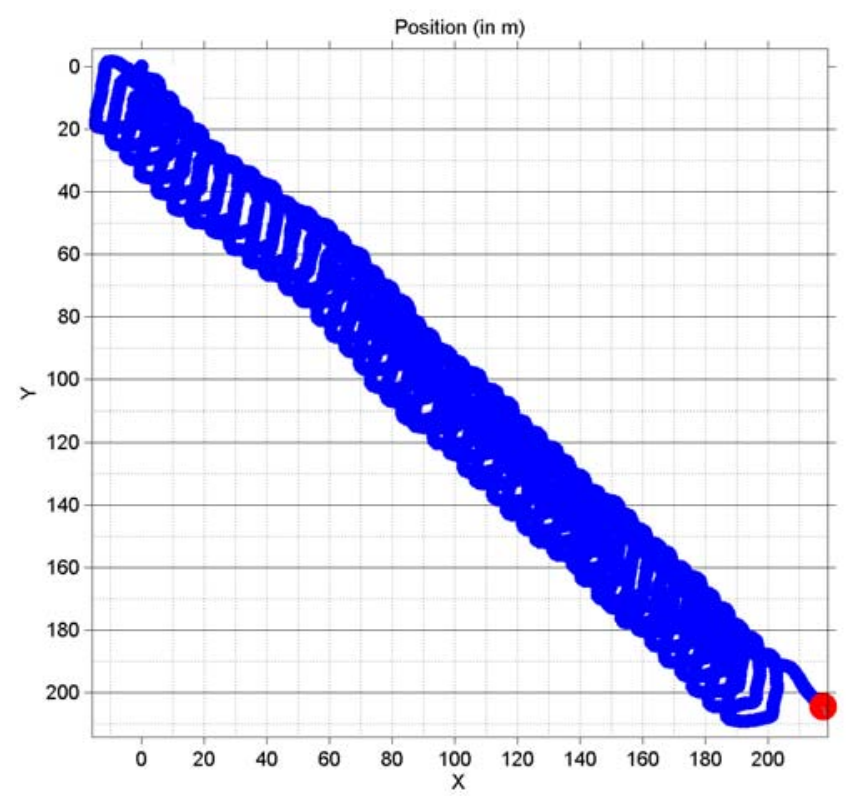

Fig. 3. Experimental results - Many laps (each being about $90 \mathrm{~m}$ long) are performed in the same loop-shaped corridor during about 45 minutes, for a total length of $3 \mathrm{~km}$. The drift, due to the altitude deviation being projected, is clearly visible.

must be kept along the $\mathrm{x}$-axis of the IMU, the velocity should be constant, and the IMU must be kept horizontal (i.e. its z-axis should be kept vertical).

2) Compute the naively reconstructed trajectory according to Equation (6) recalled below. Note $P_{r}(T)$ the final point.

$$
P_{r}(T)-P(0)=\int_{0}^{T} R_{a t t}(t) V_{v e l} \cdot d t
$$

3) Identify the pitch angle $\theta$ thanks to Equation (7).

$$
\sin (\theta)=\left\|P_{r}(T)-P(0)\right\| \cdot \frac{-1}{V_{0} \cdot T}
$$

This calibration procedure identifies the incriminated angle rather straightforward. Some of the constraints may seem hard to fulfill. However, most of them will be relaxed in Section III. 


\section{PRACTICAL CALIBRATION RESUlts AND LiMitations}

\section{A. Further theoretical investigations}

The example of Section II shows a particular case where a single angle is responsible for the non-closing of the reconstructed trajectory. We now present another viewpoint which will allow us to take some further errors in the trajectory reconstruction into account, and remove some constraints from the proposed calibration procedure. We assume that the pedestrian is performing a closed-loop trajectory with the time-varying speed in a constant direction in its own frame $\Re_{p e d}$ (forward for instance, as in Section II). The speed direction is then constant in the three frames $\Re_{p e d}, \Re_{v e l}$, and $\Re_{I M U}$ (but the speed directions do not have the same expressions in the three frames).

Again, from Equation (3), we have

$$
P(T)-P(0)=\int_{0}^{T} R_{a t t}(t) R_{\text {harmo }} V_{\text {vel }}(t) d t
$$

The speed direction being constant in the velocity frame, it can be put outside the integral sign, allowing to put the constant matrix $R_{\text {harmo }}$ outside the integral sign too. Denote $\frac{V_{v e l}}{\left\|V_{v e l}\right\|}$ the constant speed direction in the velocity frame, and $v_{\text {vel }}(t)=\left\|V_{\text {vel }}(t)\right\|$ its magnitude. Then, one gets

$$
P(T)-P(0)=\underbrace{\int_{t_{0}}^{T} v_{v e l}(t) R_{a t t}(t) d t}_{M} R_{\text {harmo }} \frac{V_{\text {vel }}}{\left\|V_{\text {vel }}\right\|}
$$

If the path followed by the pedestrian is a closed-loop trajectory, $P(T)=P(0)$, which means that $M$ has not full rank and $R_{\text {harmo }}$ must send $\frac{V_{\text {vel }}}{\left\|V_{\text {vel }}\right\|}$ in the kernel of $M$. Further, $M$ has the following property.

Proposition 1: Consider a plane closed trajectory followed with a constant speed direction in the $\Re_{\text {ped }}$ frame of reference (without spinning around that direction), starting at time $\mathrm{t}=0$ and ending at time $\mathrm{t}=\mathrm{T}$. Denote the speed magnitude

$$
v_{v e l}(t)=v_{I M U}(t)=\left\|V_{v e l}(t)\right\|
$$

Then, the $3 \times 3$ matrix

$$
M=\int_{0}^{T} v_{I M U}(t) R_{a t t}(t) d t
$$

has rank 1, and its only non-zero singular value is the traveled distance.

The proof is given in Appendix A. According to Equation (9), to close the reconstructed trajectory, and thus correct the pitch angle discussed in Section II, one simply has to find an harmonization matrix that sends $\frac{V_{v e l}}{\left\|V_{v e l}\right\|}$ in the vector space of the null singular value of $M$. For instance, a rotation matrix that sends $\frac{V_{v e l}}{\left\|V_{v e l}\right\|}$ on the direction of its projection on the plane of null singular value is a solution ${ }^{3}$.

\section{B. Dealing with experimental data}

In practice, the actual attitude and the actual velocity are almost never known, but estimated. Information about those quantities is provided through sensors, either directly or, more often, through data filtering. Denote by index $f$

\footnotetext{
${ }^{3}$ Note that this plane corresponds to the degree of freedom left by the yaw angle $\psi$ in Section II
}

the corresponding filtered quantities that are available. By integration, one gets the reconstructed position $P_{r}(t)$

$$
P_{r}(T)-P(0)=\underbrace{\int_{0}^{T} v_{v e l}^{f}(t) R_{a t t}^{f}(t) d t}_{M^{f}} R_{\text {harmo }} \frac{V_{v e l}^{f}}{\left\|V_{v e l}^{f}\right\|}
$$

As stated in Proposition 1, $M$ has a plane of null singular values and its only non-zero singular value is the traveled distance. Due to the limited bandwidth of the sensors and the filters, $M^{f}$ does not have a plane of perfectly null singular value. As a consequence, the reconstructed trajectory cannot be made perfectly closed thanks to an harmonization matrix. However, $M^{f}$ is not very different from $M$. Experiments show that the singular values of $M^{f}$ are close to those of $M$ : two are close to zero (almost identical), and a third one is close to the traveled distance. The closure error can be minimized by choosing an $R_{\text {harmo }}$ sending $\frac{V_{\text {vel }}^{f}}{\left\|V_{v e l}^{f}\right\|}$ in the vector space of the two smallest singular values. If the speed direction has been kept perfectly constant in the pedestrian frame $\Re_{\text {ped }}$, the smallest singular value is indeed the lowest closure error that can be achieved by choosing $R_{\text {harmo }}$. Experimental results are provided in Section IV.

\section{Second proposed calibration procedure}

A first calibration procedure has been proposed in Section II-B. Some constraints, potentially hard to fulfill without dedicated equipment were required to perform that procedure. The approach presented in Section III allows to remove or weaken most of them in a new calibration procedure. We now present this procedure.

1) Walk along any closed-loop trajectory in the horizontal plane with the following constraint: the speed direction must be kept along the $\mathrm{x}$-axis of the IMU.

2) Compute the matrix

$$
M_{f}=\int_{0}^{T} v_{v e l}^{f}(t) R_{a t t}^{f}(t) d t
$$

3) Identify the plane of smaller singular values of $M_{f}$ (supposed to be identical).

4) Find a rotation matrix $R_{\text {harmo }}$ which sends the speed direction $V_{d}=\frac{V_{v e l}^{f}}{\left\|V_{v e l}^{f}\right\|}$ to its projection $P\left(V_{d}\right)=$ $P\left(\frac{V_{v e l}^{f}}{\left\|V_{v e l}^{f}\right\|}\right) /\left\|\frac{V_{v e l}^{f}}{\left\|V_{v e l}^{f}\right\|}\right\|$ in that plane of singular values, for instance thanks to Olinde Rodrigues' formula.

$$
\begin{aligned}
N= & \frac{P\left(V_{d}\right) \times V_{d}}{\left\|P\left(V_{d}\right) \times V_{d}\right\|}=\left(\begin{array}{c}
N_{1} \\
N_{2} \\
N_{3}
\end{array}\right) \\
\alpha= & a \cos \left(V_{d}^{T} P\left(V_{d}\right)\right) \\
R_{\text {harmo }}= & \cos (\alpha) I_{3}+(1-\cos (\alpha)) N N^{T} \\
& +\sin (\alpha)\left(\begin{array}{ccc}
0 & -N_{3} & N_{2} \\
N_{3} & 0 & -N_{1} \\
-N_{2} & N_{1} & 0
\end{array}\right)
\end{aligned}
$$

Two of the requirements of the first calibration procedure have been removed: keeping the speed constant and the $\mathrm{z}$ axis vertical. The required trajectory is now simply the one naturally performed by any pedestrian walking, for instance, in a loop-shaped corridor. This allows the system to be calibrated very easily in the field by any operator. 

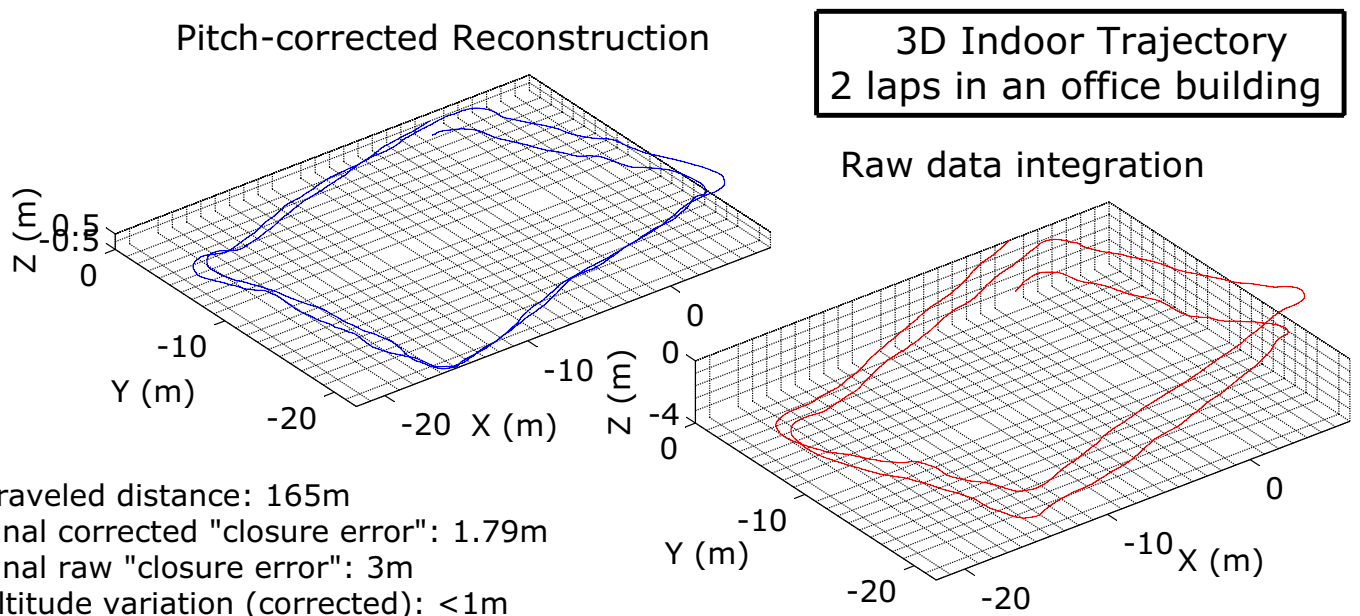

Altitude variation (raw): $3.5 \mathrm{~m}$

Pitch angle determined: $0.8^{\circ}$

Fig. 4. Experimental data - The pitch-angle has been corrected. The trajectory does not drift downward anymore.

\section{EXPERIMENTAL VALIDATION}

In order to evaluate the proposed calibration method, a system following the modular design in two separate modules for speed and attitude estimations has been implemented. The attitude is classically estimated by use of inertial sensors (see [3], and the references therein) while the velocity estimate is provided through a set of spatially distributed magnetometers as proposed in [9]. Briefly, the magnetic field dynamics in the velocity frame writes

$$
\dot{M}_{v e l}=-\Omega \times M_{v e l}+J_{v e l}(M) V_{v e l}
$$

where $M_{v e l}$ denotes the magnetic field in the velocity frame. It is measured by the magnetometers. $\Omega$, the rate of turn between the velocity frame and the inertial frame of reference $\Re_{I}$, is given by a gyrometer. $J_{v e l}(M)$ denotes the Jacobian of the magnetic field expressed in the velocity frame, i.e. it represents the local spatial variations of the magnetic field. It is obtained from the measurements of the spatially distributed magnetometers set through a simple finite difference scheme. Finally, $V_{v e l}$ is the sought after velocity. Thus, the measurement system consists of a set of distributed magnetometers and an IMU. Both of them are attached to a single rigid board. This board is carried by the pedestrian whose trajectory is reconstructed. The system is detailed on Figure 5, and illustrated in Figure 6, where it is carried by a pedestrian.

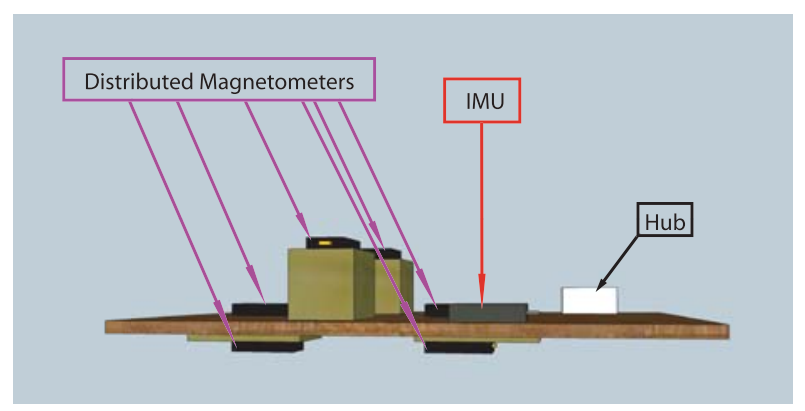

Fig. 5. The experimental system consists of an inertial measurement unit to estimate the attitude, and of a set of spatially distributed magnetometers to separately estimate the velocity.
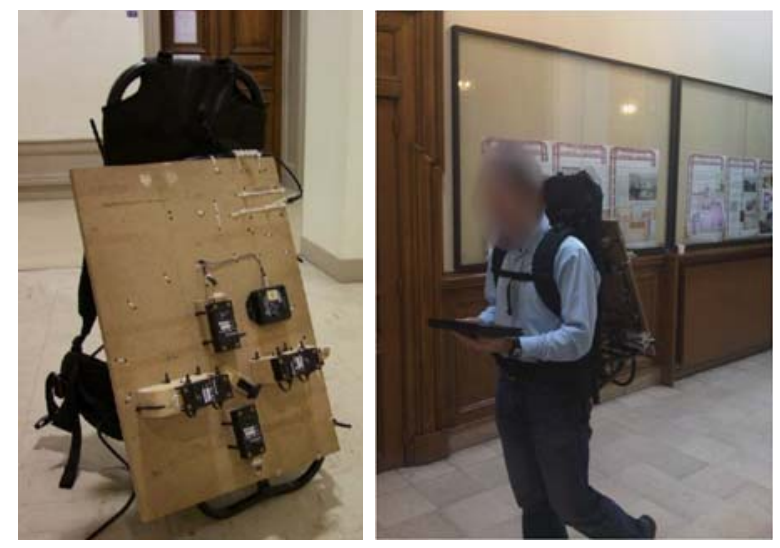

Fig. 6. The experimental system in action.

Figure 4 shows some detailed experimental results. The trajectory is reconstructed after walking two laps along a loopshaped corridor in an office building with the described pedestrian navigation system in the back. The red curve represents the (raw) trajectory obtained without taking the harmonization matrix into account, whereas the blue one is obtained from the same data but with insertion of the harmonization matrix (the pitch rotation angle is about 0.8 degrees). The altitude drift is canceled.

Finally, we show, in Figure 7, an example of reconstructed trajectory that can be achieved by the system once the harmonization matrix has been found. The trajectory is projected on a 2-D map of the building. After a $220 \mathrm{~m}$ walk in two levels of an office building, the final error is approximatively $5 \mathrm{~m}$. (The typical error of this system is about $4-5 \%$ of the traveled distance.)

\section{CONCLUSION AND FUTURE WORK}

In this paper, the harmonization errors between a velocimeter and an attitude sensor mounted on the same rigid body has been analyzed. It has been shown that a single pitch angle harmonization error is responsible for altering the shape of the reconstructed trajectory, e.g. by making a closed trajectory opened. A simple procedure to estimate this angle has been 


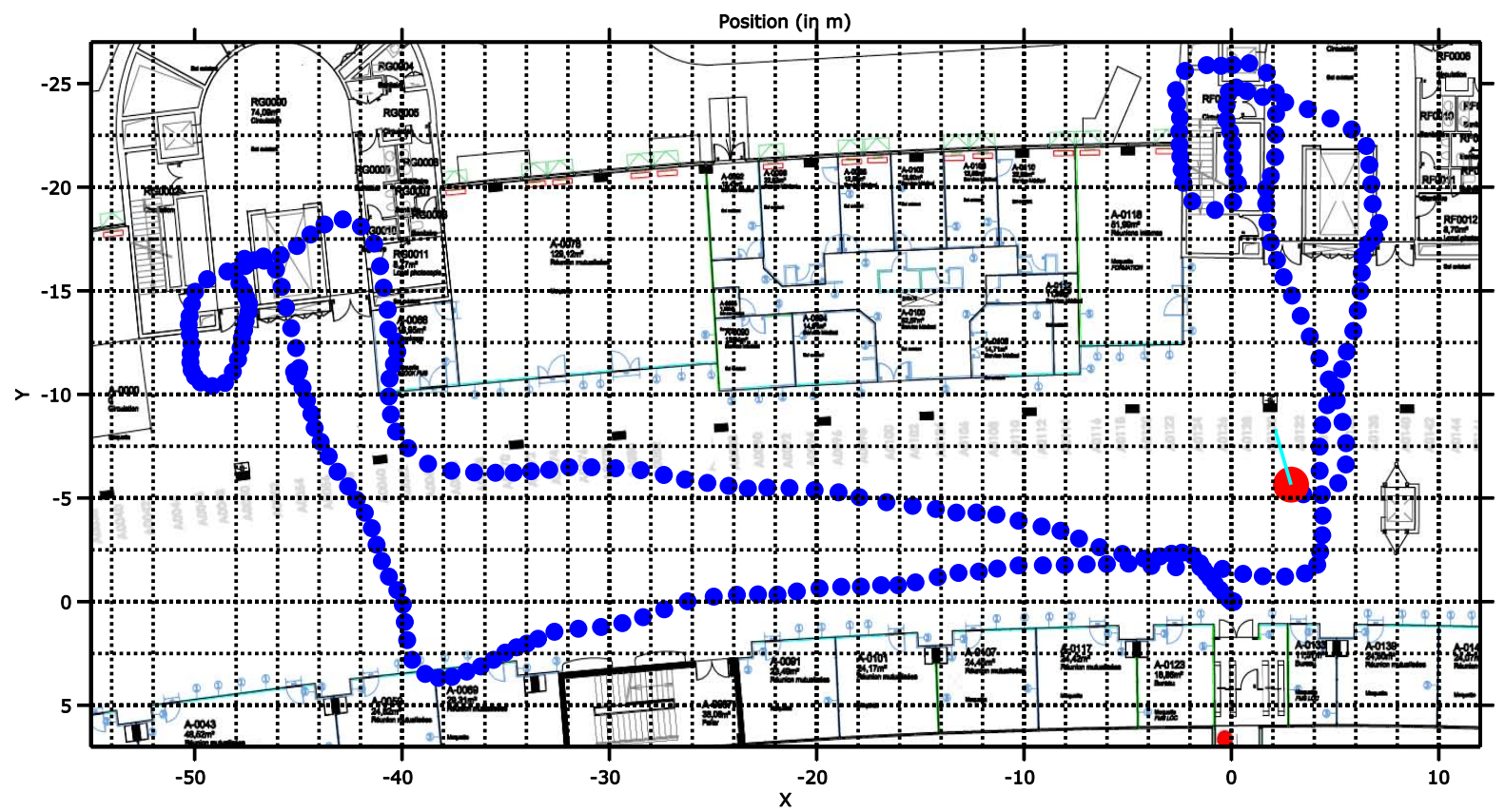

Fig. 7. Example of reconstructed trajectory. The pedestrian starts at the entrance of the building after passing the doors, walks through the huge hall, walk the stairs one level up. Then, he takes a bridge over the hall, go along the offices, takes another bridge over the hall, goes down the steps, and goes back to its starting point. The final error is about 5 meters after walking $220 \mathrm{~m}$ in 4 minutes and 19 seconds.

proposed, which can easily be performed on the field. Then, a more general formulation being able to account for some further imperfections due to the filtering of measurements has been proposed. Further developments will include the identification of less detrimental effects on the orientation of the trajectory.

\section{ACKNOWLEDGMENTS}

This work was partially funded by the SYSTEM@TIC PARIS-REGION Cluster in the frame of project LOCINDOOR.

\section{REFERENCES}

[1] S. Esquivel, F. Woelk, and R. Koch. Calibration of a multi-camera rig from non-overlapping views. Lecture Notes in Computer Science (including subseries Lecture Notes in Artificial Intelligence and Lecture Notes in Bioinformatics), 4713 LNCS:82-91, 2007.

[2] D. Gebre-Egziabher, G. Elkaim, J. Powell, and B. Parkinson. A nonlinear, two-step estimation algorithm for calibrating solid-state strapdown magnetometers. In 8th International St. Petersburg Conference on Navigation Systems (IEEE/AIAA), May 2001.

[3] T. Hamel and R. Mahony. Image based visual servo-control for a class of aerial robotic systems. Automatica, 43(11):1975-1983, 2007.

[4] J. Hesch, F. Mirzaei, G. Mariottini, and S. Roumeliotis. A laser-aided inertial navigation system (1-ins) for human localization in unknown indoor environments. In Proc. of the 2010 IEEE International Conference on Robotics and Automation (ICRA), pages 5376-5382, 2010.

[5] F. Mirzaei and S. Roumeliotis. A kalman filter-based algorithm for imucamera calibration: Observability analysis and performance evaluation. IEEE Transactions on Robotics, 24(5):1143-1156, 2008.

[6] M. A. Penna. Camera calibration: A quick and easy way to determine the scale factor. IEEE Transactions on Pattern Analysis and Machine Intelligence, 13(12):1240-1245, 1991.

[7] C. Taylor. Long-term accuracy of camera and imu fusion-based navigation systems. In Proc. of the Conference of Institute of Navigation - International Technical Meeting, ITM 2009, volume 1, pages 93-101, 2009 .
[8] R. Tillett. A calibration system for vision-guided agricultural robots. Journal of Agricultural Engineering Research, 42(4):267-273, 1989.

[9] D. Vissière, A.-P. Martin, and N. Petit. Using spatially distributed magnetometers to increase IMU-based velocity estimation in perturbed areas. In Proc. of the 46th IEEE Conf. on Decision and Control, 2007.

\section{APPENDIX A}

Proof: First assume that the IMU has its $\mathrm{x}$ and $\mathrm{y}$ axis in the horizontal plane. The attitude is then a simple rotation around the vertical $\mathrm{z}$ axis defined by a single angle $\alpha(t)$ as already defined in Equation (4). For any constant speed direction

$$
\frac{V_{I M U}}{\left\|V_{I M U}\right\|}=\left(\begin{array}{c}
v_{x} \\
v_{y} \\
0
\end{array}\right)
$$

chosen in the horizontal plane of the IMU-frame, as long as the starting point and the end point are identical, we get

$$
0=\left(\int_{0}^{T} v_{I M U}(t)\left(\begin{array}{ccc}
c(\alpha) & -s(\alpha) & 0 \\
s(\alpha) & c(\alpha) & 0 \\
0 & 0 & 1
\end{array}\right) d t\right)\left(\begin{array}{c}
v_{x} \\
v_{y} \\
0
\end{array}\right)
$$

where $v_{I M U}(t)=\left\|V_{I M U}(t)\right\|$. Equation (16) gives a linear equation in $\left(\begin{array}{ll}v_{x} & v_{y}\end{array}\right)^{T}$

$$
\underbrace{\left(\begin{array}{ll}
\int_{0}^{T} v_{I M U}(t) c(\alpha) & -\int_{0}^{T} v_{I M U}(t) s(\alpha) \\
\int_{0}^{T} v_{I M U}(t) s(\alpha) & \int_{0}^{T} v_{I M U}(t) c(\alpha)
\end{array}\right)}_{A}\left(\begin{array}{c}
v_{x} \\
v_{y}
\end{array}\right)=\left(\begin{array}{c}
0 \\
0
\end{array}\right)
$$

As $\left(v_{x}, v_{y}\right) \neq(0,0)$, we deduce that $A$ has not full rank. Yet,

$$
\underbrace{\operatorname{det}(A)}_{=0}=\left(\int_{0}^{T} v_{I M U}(t) c(\alpha) d t\right)^{2}+\left(\int_{0}^{T} v_{I M U}(t) s(\alpha) d t\right)^{2}
$$


which is null if and only if both squared terms are null, i.e.

$$
\int_{0}^{T} v_{I M U}(t) c(\alpha) d t=0 \text { and } \int_{0}^{T} v_{I M U}(t) s(\alpha) d t=0
$$

Replacing those quantities in Equation (16) yields

$$
M=\left(\begin{array}{ccc}
0 & 0 & 0 \\
0 & 0 & 0 \\
0 & 0 & \int_{0}^{T} v_{I M U}(t) d t
\end{array}\right)
$$

which shows that $M$ is of rank 1 .

The starting frame on the right side of $R_{\alpha}$ and the arrival frame on the left side of $R_{\alpha}$ can be rotated by some orthogonal matrices $P$ and $Q$, taking into account the fact that the IMU is not horizontal anymore $(P)$ and that the coordinate frame of the inertial frame is arbitrary $(Q)$.
We can get rid off $P$ and $Q$ under the integral sign since they are constant. This yields

$$
\begin{aligned}
M & =\int_{0}^{T} v_{I M U}(t) Q^{T}\left(\begin{array}{ccc}
\cos (\alpha) & -\sin (\alpha) & 0 \\
\sin (\alpha) & \cos (\alpha) & 0 \\
0 & 0 & 1
\end{array}\right) P d t \\
& =Q^{T}\left(\begin{array}{ccc}
0 & 0 & 0 \\
0 & 0 & 0 \\
0 & 0 & \underbrace{\int_{0}^{T} v_{I M U}(t) d t}_{\text {traveled distance }}
\end{array}\right) P
\end{aligned}
$$

which is the sought after singular value decomposition of $M$ with the traveled distance as only non-zero singular value. 